\title{
A Six-order Variant of Newton's Method for Solving Nonlinear Equations
}

\author{
Manoj Kumar Singh \\ Department of Mathematics, Faculty of Science \\ Banaras Hindu University \\ Varanasi, India \\ e-mail:manoj07777@gmail.com
}

(Received: 30 October 2009; revised: 05 December 2009; accepted: 10 December 2009; published online: 30 December 2009)

\begin{abstract}
A new variant of Newton's method based on contra harmonic mean has been developed and its convergence properties have been discussed. The order of convergence of the proposed method is six. Starting with a suitably chosen $x_{0}$, the method generates a sequence of iterates converging to the root. The convergence analysis is provided to establish its sixth order of convergence. In terms of computational cost, it requires evaluations of only two functions and two first order derivatives per iteration. This implies that efficiency index of our method is 1.5651. The proposed method is comparable with the methods of Parhi, and Gupta [15] and that of Kou and Li [8]. It does not require the evaluation of the second order derivative of the given function as required in the family of Chebyshev-Halley type methods. The efficiency of the method is tested on a number of numerical examples. It is observed that our method takes lesser number of iterations than Newton's method and the other third order variants of Newton's method. In comparison with the sixth order methods, it behaves either similarly or better for the examples considered.
\end{abstract}

Key words: Newton's method, iteration function, order of convergence, function evaluations, efficiency index

\section{INTRODUCTION}

In Science and Engineering, many of the nonlinear and transcendental problems of the form $f(x)=0$, are complex in nature. Since it is not always possible to obtain its exact solution by the usual algebraic process, the numerical iterative methods such as Newton, secant methods are often used to obtain the approximate solution of such problems. Though these methods are very effective, there are some limitations that they do not give the result as fast as one wants, and take several iterations. There are many methods developed on the improvement of quadratically convergent Newton's method, so as to get a superior convergence order than Newton. This paper is concerned with the iterative methods for finding a simple root $\alpha$, i.e. $f(\alpha)=0$, and $f^{\prime}(\alpha) \neq 0$ of $f(x)=0$, where $f: R \rightarrow R$, be the continuously differentiable real function. The symbols used here have their usual meanings.

We consider the problem of finding a real zero of a function $f: I \subset R \rightarrow R$. This zero can be determined as a fixed point of some iteration function $g$ by means of the one-point iteration method

$$
x_{n+1}=g\left(x_{n}\right), \quad n=0,1 \ldots
$$

where $x_{0}$ is the starting value. The best known and the most widely used example of these types of methods is the classical Newton's method given by

$$
x_{n+1}=x_{n}-\frac{f\left(x_{n}\right)}{f^{\prime}\left(x_{n}\right)}, \quad n=0,1 \ldots
$$

It converges quadratically to simple zeros and linearly to multiple zeros. In the literature, some of its modifications have been introduced in order to accelerate it or to get a method with a higher order of convergence. If we consider the definition of efficiency index as $p^{1 / m}$, where $p$ is the order of the method and $m$ is the number of functions evaluation required by the method (units of work per iteration) then the efficiency index of this method is 1.414. A number of ways are considered by many researchers to improve the local order of convergence of Newton's 
method at the expense of additional evaluations of functions, derivatives and changes in the points of iterations.

All these modifications are in the direction of increasing the local order of convergence with the view of increasing their efficiency indices. For example, the method developed by Weerakoon [18], called as trapezoidal Newton's or arithmetic mean Newton's method, suggests for some other variants of Newton's method. Frontini and Sormani [3] developed new modifications of Newton's method to produce the iterative method with the order of convergence of three and efficiency index of 1.442. With the same efficiency index, Ozban [14] also gave some new variants of Newton's method, and Chen [2] described some new iterative formulae having third order convergence.

Ostrowski [12] developed both third and fourth order methods, each requiring evaluations of two functions and one derivative per iteration. Maheshwari [11] developed a fourth order iterative method for solving non linear equations. Recently a number of sixth order methods have also been appearing as the extensions of the abovementioned methods to solve $f(x)=0$. Sharma and Guha [16] developed a one parameter family of sixth order methods based on Ostrowski fourth order multipoint method. Each family required three evaluations of the given function and one evaluation of the derivative per iteration. Chun [1] presented a one parameter family of variants of Jarratt's fourth order method for solving nonlinear equations. It shows there that the order of convergence of each family member is improved from order four to six even though it adds one evaluation of the function at the point iterated by Jarratt's method per iteration. Kou et al. [6-9] presented a family of new variants of Chebyshev-Halley methods and also an improvement of Jarratt method. These new methods have sixth order of convergence although they only add one evaluation of the function at the point iterated by Chebyshev-Halley method and Jarratt method. Parhi and Gupta, [15] developed a sixth order method for nonlinear equations, by extending a third order method of Weerakoon and Fernando [18], requires evaluations of two functions and two first derivative per iteration.

Our method also requires evaluations of two functions and two first derivatives per iteration, is comparable with the methods of Kou and Li [8] and that of Parhi and Gupta [15]. It does not require the evaluation of the second order derivative of the given function as required in ChebyshevHalley type methods $[6,7]$. The efficiency of the method is tested on a number of numerical examples. It is observed that the proposed method takes a lower number of iterations than taken by Newton's method and the other third order variant of Newton's method. In comparison with the other sixth order methods, it behaves either similarly or better for the examples considered.

\section{DEFINITIONS}

Definition II.1. See [5, 13, 17]

If the sequence $\left\{x_{n} / n \geq 0\right\}$ tends to a limit $\alpha$ in such a way that

$$
\lim _{x_{n \rightarrow \alpha}} \frac{x_{n+1}-\alpha}{\left(x_{n}-\alpha\right)^{p}}=C,
$$

for some $C \neq 0$ and $p \geq 1$, then the order of convergence of the sequence is said to be $p$, and $C$ is known as asymptotic error constant.

When $p=1$, the convergence is linear, and it is called first order convergence.

While for $p=2$ and $p=3$ the sequence is said to converge quadratically and cubically, respectively.

The value of $p$ is called the order of convergence of the method which produces the sequence $\left\{x_{n} / n \geq 0\right\}$. Let $e_{n}=x_{n}-\alpha$, then the relation $e_{n+1}=C e_{n}^{p}+O\left(e_{n}^{p+1}\right)$ is called the error equation for the method, $p$ being the order of convergence.

\section{Definition II.2. See $[13,17]$}

Efficiency index is simply defined as $p^{1 / m}$ where $p$ is the order of the method and $m$ is the number of functions evaluations required by the method (units of work per iteration).

Therefore the efficiency index of Newton's method is 1.414 and that of third order method is 1.442 and efficiency index of method proposed by me is 1.5651 .

\section{DESCRIPTION OF METHODS}

Let $\alpha$ be a simple zero of a sufficiently differentiable function $f$ and consider the numerical solution of the equation $f(\quad)=0$, then it can be written as

$$
f(x)=f\left(x_{n}\right)+\int_{x_{n}}^{x} f^{\prime}(t) d t .
$$

Suppose, we interpolate $f^{\prime}$ on the interval $\left[x_{n}, \quad\right]$ by constant $f^{\prime}\left(x_{n}\right)$, then $\left(x-x_{n}\right) f^{\prime}\left(x_{n}\right)$ provides an approximation for the integral in (3) and by taking $x=\alpha$, we obtain

$$
0 \approx f\left(x_{n}\right)+\left(\alpha-x_{n}\right) f^{\prime}\left(x_{n}\right),
$$

and hence, a new approximation $x_{n+1}$ to $\alpha$ is given by

$$
x_{n+1}=x_{n}-\frac{f\left(x_{n}\right)}{f^{\prime}\left(x_{n}\right)} \quad n=0,1, \ldots
$$


which is Newton's method for $n=0,1 \ldots$. On the other hand, if we approximate the indefinite integral in (3) by the trapezoidal rule and take $x=\alpha$, we obtain

$$
0 \approx f\left(x_{n}\right)+1 / 2\left(\alpha-x_{n}\right)\left(f^{\prime}\left(x_{n}\right)+f^{\prime}(\alpha)\right),
$$

and therefore, a new approximation $x_{n+1}$ to $\alpha$ is given by

$$
x_{n+1}=x_{n}-\frac{2 f\left(x_{n}\right)}{f^{\prime}\left(x_{n}\right)+f^{\prime}\left(x_{n+1}\right)} .
$$

If the $(n+1)$ value of Newton's method is used on the right-hand side of the above equation to overcome the implicity problem, then

$$
x_{n+1}=x_{n}-\frac{2 f\left(x_{n}\right)}{f^{\prime}\left(x_{n}\right)+f^{\prime}\left(z_{n+1}\right)},
$$

where:

$$
z_{n+1}=x_{n}-f\left(x_{n}\right) / f^{\prime}\left(x_{n}\right)
$$

is obtained which is, for $n=0,1,2, \ldots$, the trapezoidal Newton's method of Weerakoon [18].

Let us rewrite Eq. (4) as

$$
x_{n+1}=x_{n}-\frac{f\left(x_{n}\right)}{\left(f^{\prime}\left(x_{n}\right)+f^{\prime}\left(z_{n+1}\right)\right) / 2}, \quad n=0,1,2 \ldots
$$

and further to re-write as

$$
z_{n}=x_{n}-\frac{f\left(x_{n}\right)}{\left(f^{\prime}\left(x_{n}\right)+f^{\prime}\left(y_{n}\right)\right) / 2}, \quad n=0,1,2 \ldots
$$

So this variant of Newton's method can be viewed as obtained by using arithmetic mean of $f^{\prime}\left(x_{n}\right)$ and $f^{\prime}\left(y_{n}\right)$ instead of $f^{\prime}\left(x_{n}\right)$ in Newton s method defined by (1), which was called as arithmetic mean Newton's method.

\section{New variant of Newton's method}

The contra-harmonic mean, "c", of any two real numbers " $a$ " and " $b$ " is defined as

$$
c=\frac{\left(a^{2}+b^{2}\right)}{(a+b)} .
$$

In (5), if we use the Contra harmonic mean instead of the arithmetic mean we get

$$
\begin{gathered}
y_{n}=x_{n}-\frac{f\left(x_{n}\right)}{f^{\prime}\left(x_{n}\right)}, \\
z_{n}=x_{n}-\frac{f\left(x_{n}\right)\left(f^{\prime}\left(x_{n}\right)+f^{\prime}\left(y_{n}\right)\right)}{f^{\prime}\left(x_{n}\right)^{2}+f^{\prime}\left(y_{n}\right)^{2}}, \quad n=0,1,2 \ldots
\end{gathered}
$$

Again

$$
x_{n+1}=z_{n}-\frac{f\left(z_{n}\right)}{f^{\prime}\left(z_{n}\right)}, \quad n=0,1, \ldots
$$

Now using the linear interpolation on two points $\left(x_{n}, f^{\prime}\left(x_{n}\right)\right)$ and $\left(y_{n}, f^{\prime}\left(y_{n}\right)\right)$ we get

$$
f^{\prime}(x) \approx \frac{x-x_{n}}{y_{n}-x_{n}} f^{\prime}\left(y_{n}\right)+\frac{x-y_{n}}{x_{n}-y_{n}} f^{\prime}\left(x_{n}\right) .
$$

Thus an approximation to $f^{\prime}\left(z_{n}\right)$ is given by

$$
\begin{gathered}
f^{\prime}\left(z_{n}\right) \approx \frac{z_{n}-x_{n}}{y_{n}-x_{n}} f^{\prime}\left(y_{n}\right)+\frac{z_{n}-y_{n}}{x_{n}-y_{n}} f^{\prime}\left(x_{n}\right) \\
f^{\prime}\left(z_{n}\right) \approx \frac{2 f^{\prime}\left(x_{n}\right) f^{\prime}\left(y_{n}\right)^{2}}{f^{\prime}\left(x_{n}\right)^{2}+f^{\prime}\left(y_{n}\right)^{2}}
\end{gathered}
$$

From Eq. (9) and Eq. (11) our proposed method can be written as

$$
\begin{gathered}
y_{n}=x_{n}-\frac{f\left(x_{n}\right)}{f^{\prime}\left(x_{n}\right)} \\
z_{n}=x_{n}-\frac{f\left(x_{n}\right)\left(f^{\prime}\left(x_{n}\right)+f^{\prime}\left(y_{n}\right)\right)}{f^{\prime}\left(x_{n}\right)^{2}+f^{\prime}\left(y_{n}\right)^{2}}, \\
x_{n+1}=z_{n}-\frac{f\left(z_{n}\right)\left(f^{\prime}\left(x_{n}\right)^{2}+f^{\prime}\left(y_{n}\right)^{2}\right)}{2 f^{\prime}\left(x_{n}\right) f^{\prime}\left(y_{n}\right)} .
\end{gathered}
$$

Clearly this method requires evaluations of two functions $f$ and two derivative $f^{\prime}$ and no second order derivative $f^{\prime \prime}$ of $f$.

\section{CONVERGENCE ANALYSIS}

\section{Theorem IV.1.}

Let $\alpha \in I$ be a simple zero of a sufficiently differentiable function $f: I \subset R \rightarrow R$ for an open interval $I$. If $x_{0}$ is sufficiently close to $\alpha$, then the method defined by (12) has six-th order convergence.

\section{Proof.}

Let $\alpha \in I$ be a simple zero of $f$. Since $f$ is sufficiently differentiable, by expanding $f\left(x_{n}\right)$ and $f^{\prime}\left(x_{n}\right)$ about $\alpha$ we get

$$
f\left(x_{n}\right)=f\left(\alpha+e_{n}\right)=f^{\prime}(\alpha) e_{n}+f^{\prime \prime}(\alpha) \frac{e_{n}^{2}}{2 !} f^{\prime \prime \prime}(\alpha) \frac{e_{n}^{3}}{3 !}+O\left(e_{n}^{4}\right),
$$




$$
f\left(x_{n}\right)=f^{\prime}(\alpha)\left[e_{n}+C_{2} e_{n}^{2}+C_{3} e_{n}^{3}+O\left(e_{n}^{4}\right)\right],
$$

where:

$$
C_{j}=(1 / j !) f^{j}(\alpha) / f(\alpha) \text {, }
$$

$$
\begin{gathered}
f^{\prime}\left(x_{n}\right)=f^{\prime}\left(\alpha+e_{n}\right)=f^{\prime}(\alpha)+f^{\prime \prime}(\alpha) e_{n}+f^{\prime \prime \prime}(\alpha) \frac{e_{n}^{2}}{3 !}+O\left(e_{n}^{4}\right)= \\
f^{\prime}(\alpha)\left[1+2 C_{2} e_{n}+3 C_{3} e_{n}^{2}+4 C_{4} e_{n}^{3}+O\left(e_{n}^{4}\right)\right], \\
\frac{f\left(x_{n}\right)}{f^{\prime}\left(x_{n}\right)}=\frac{\left[e_{n}+C_{2} e_{n}^{2}+C_{3} e_{n}^{3}+O\left(e_{n}^{4}\right)\right]}{\left[1+2 C_{2} e_{n}+3 C_{3} e_{n}^{2}+4 C_{4} e_{n}^{3}+O\left(e_{n}^{4}\right)\right]}=\left[e_{n}-C_{2} e_{n}^{2}+\left(2 C_{2}^{2}-2 C_{3}\right) e_{n}^{3}+O\left(e_{n}^{4}\right)\right]
\end{gathered}
$$

Newton's method is

$$
\begin{gathered}
y_{n}=x_{n}-\frac{f\left(x_{n}\right)}{f^{\prime}\left(x_{n}\right)} \\
y_{n}=\alpha+C_{2} e_{n}^{2}+\left(2 C_{3}-2 C_{2}^{2}\right) e_{n}^{3}+O\left(e_{n}^{4}\right)
\end{gathered}
$$

That is Newton's method converges quadratically. Again we have:

$$
\begin{gathered}
y_{n}=\alpha+C_{2} e_{n}^{2}+\left(2 C_{3}-2 C_{2}^{2}\right) e_{n}^{3}+O\left(e_{n}^{4}\right), \\
f^{\prime}\left(y_{n}\right)=f^{\prime}(\alpha)\left[1+2 C_{2}^{2} e_{n}^{2}+4 C_{2}\left(C_{3}-C_{2}^{2}\right) e_{n}^{3}+O\left(e_{n}^{4}\right)\right], \\
f^{\prime}\left(x_{n}\right)^{2}=f^{\prime}(\alpha)^{2}\left[1+4 C_{2} e_{n}+\left(4 C_{2}^{2}+6 C_{3}\right) e_{n}^{2}+\left(8 C_{4}+12 C_{2} C_{3}\right) e_{n}^{3}+O\left(e_{n}^{4}\right)\right] .
\end{gathered}
$$

Again

$$
f^{\prime}\left(y_{n}\right)^{2}=f^{\prime}(\alpha)^{2}\left[1+4 C_{2}^{2} e_{n}^{2}+\left(8 C_{2} C_{3}-8 C_{2}^{3}\right) e_{n}^{3}+O\left(e_{n}^{4}\right)\right]
$$

Adding $f^{\prime}\left(x_{n}\right)$ and $f^{\prime}\left(y_{n}\right)$ we get

$$
\begin{gathered}
f^{\prime}\left(x_{n}\right)+f^{\prime}\left(y_{n}\right)=2 f^{\prime}(\alpha)\left[1+C_{2} e_{n}+\left(C_{2}^{2}+\frac{3}{2} C_{3}\right) e_{n}^{2}+2\left(C_{2} C_{3}-C_{2}^{3}+C_{4}\right) e_{n}^{3}+O\left(e_{n}^{4}\right)\right], \\
f\left(x_{n}\right)\left(f^{\prime}\left(x_{n}\right)+f^{\prime}\left(y_{n}\right)\right)=2 f^{\prime}(\alpha)^{2}\left[e_{n}+2 C_{2} e_{n}^{2}+\left(2 C_{2}^{2}+\frac{5}{2} C_{3}\right) e_{n}^{3}+O\left(e_{n}^{4}\right)\right] .
\end{gathered}
$$

Again adding $f^{\prime}\left(x_{n}\right)^{2}$ and $f^{\prime}\left(y_{n}\right)^{2}$ we have:

$$
f^{\prime}\left(x_{n}\right)^{2}+f^{\prime}\left(y_{n}\right)^{2}=2 f^{\prime}(\alpha)^{2}\left[1+2 C_{2} e_{n}+\left(4 C_{2}^{2}+3 C_{3}\right) e_{n}^{2}+\left(10 C_{2} C_{3}-4 C_{2}^{3}+4 C_{4}\right) e_{n}^{3}+O\left(e_{n}^{4}\right)\right] .
$$

Our method is:

$$
z_{n}=x_{n}-\frac{f\left(x_{n}\right)\left(f^{\prime}\left(x_{n}\right)+f^{\prime}\left(y_{n}\right)\right)}{f^{\prime}\left(x_{n}\right)^{2}+f^{\prime}\left(y_{n}\right)^{2}}, \quad n=0,1,2, \ldots
$$

where:

$$
y_{n}=x_{n}-\frac{f\left(x_{n}\right)}{f^{\prime}\left(x_{n}\right)},
$$

and

$$
x_{n+1}=z_{n}-\frac{f\left(z_{n}\right)}{f^{\prime}\left(z_{n}\right)} .
$$




$$
\begin{aligned}
& z_{n}=x_{n}-\frac{f\left(x_{n}\right)\left(f^{\prime}\left(x_{n}\right)+f^{\prime}\left(y_{n}\right)\right)}{f^{\prime}\left(x_{n}\right)^{2}+f^{\prime}\left(y_{n}\right)^{2}} \\
& =\frac{2 f^{\prime}(\alpha)^{2}\left[e_{n}+2 C_{2} e_{n}^{2}+\left(2 C_{2}^{2}+\frac{5}{2} C_{3}\right) e_{n}^{3}+O\left(e_{n}^{4}\right)\right]}{2 f^{\prime}(\alpha)^{2}\left[1+2 C_{2} e_{n}+\left(4 C_{2}^{2}+3 C_{3}\right) e_{n}^{2}+\left(10 C_{2} C_{3}-4 C_{2}^{3}+4 C_{4}\right) e_{n}^{3}+O\left(e_{n}^{4}\right)\right]} \\
& =\frac{\left[e_{n}+2 C_{2} e_{n}^{2}+\left(2 C_{2}^{2}+\frac{5}{2} C_{3}\right) e_{n}^{3}+O\left(e_{n}^{4}\right)\right]}{\left[1+2 C_{2} e_{n}+\left(4 C_{2}^{2}+3 C_{3}\right) e_{n}^{2}+\left(10 C_{2} C_{3}-4 C_{2}^{3}+4 C_{4}\right) e_{n}^{3}+O\left(e_{n}^{4}\right)\right]} \\
& =\left[e_{n}+2 C_{2} e_{n}^{2}+\left(2 C_{2}^{2}+\frac{5}{2} C_{3}\right) e_{n}^{3}+O\left(e_{n}^{4}\right)\right]\left[1-2 C_{2} e_{n}-3 C_{3} e_{n}^{2}-\left(-2 C_{2} C_{3}-12 C_{2}^{3}+4 C_{4}\right) e_{n}^{3}+O\left(e_{n}^{4}\right)\right] \\
& =e_{n}-\left(2 C_{2}^{2}+\frac{C_{3}}{2}\right) e_{n}^{3}+O\left(e_{n}^{4}\right)=z_{n}=\alpha+\left(2 C_{2}^{2}+\frac{C_{3}}{2}\right) e_{n}^{3}+O\left(e_{n}^{4}\right), \\
& f^{\prime}\left(z_{n}\right) \approx \frac{2 f^{\prime}\left(x_{n}\right) f^{\prime}\left(y_{n}\right)^{2}}{f^{\prime}\left(x_{n}\right)^{2}+f^{\prime}\left(y_{n}\right)^{2}} .
\end{aligned}
$$

On solving, we have:

$$
f^{\prime}\left(z_{n}\right) \approx f^{\prime}(\alpha)\left[1+A e_{n}^{3}+O\left(e_{n}^{4}\right)\right]
$$

where $A$ is some constant.

Hence, from:

$$
\begin{gathered}
x_{n+1}=z_{n}-\frac{f\left(z_{n}\right)}{f^{\prime}\left(z_{n}\right)}, \\
x_{n+1}=\alpha+\left(2 C_{2}^{2}+\frac{C_{3}}{2}\right) e_{n}^{3}+O\left(e_{n}^{4}\right)-\frac{f\left(\alpha+\left(2 C_{2}^{2}+\frac{C_{3}}{2}\right) e_{n}^{3}+O\left(e_{n}^{4}\right)\right)}{f^{\prime}(\alpha)\left[1+A e_{n}^{3}+O\left(e_{n}^{4}\right)\right]}=\alpha+\left(2 C_{2}^{2}+\frac{C_{3}}{2}\right) e_{n}^{3}+O\left(e_{n}^{4}\right)+ \\
f(\alpha)+\left\{\left(2 C_{2}^{2}+\frac{C_{3}}{2}\right) e_{n}^{3}+O\left(e_{n}^{4}\right)\right\} f^{\prime}(\alpha)+\left\{\left(2 C_{2}^{2}+\frac{C_{3}}{2}\right)^{2} e_{n}^{6}+O\left(e_{n}^{7}\right)\right\} \frac{f^{\prime \prime}(\alpha)}{2 !} \\
-\frac{f^{\prime}(\alpha)\left[1+A e_{n}^{3}+O\left(e_{n}^{4}\right)\right]}{f^{\prime}}= \\
=\alpha+\left(2 C_{2}^{2}+\frac{C_{3}}{2}\right) e_{n}^{3}+O\left(e_{n}^{4}\right)-\frac{f^{\prime}(\alpha)\left\{\left(2 C_{2}^{2}+\frac{C_{3}}{2}\right) e_{n}^{3}+O\left(e_{n}^{4}\right)\right\}+\left\{\left(2 C_{2}^{2}+\frac{C_{3}}{2}\right)^{2} e_{n}^{6}+O\left(e_{n}^{7}\right)\right\} C_{2}}{f^{\prime}(\alpha)\left[1+A e_{n}^{3}+O\left(e_{n}^{4}\right)\right]} .
\end{gathered}
$$

On solving, we have

$$
x_{n+1}=\alpha+A e_{n}^{6}+O\left(e_{n}^{7}\right), \quad e_{n+1}=A e_{n}^{6}+O\left(e_{n}^{7}\right) .
$$

Hence the proposed method defined by (12) has sixth order convergence. 


\section{NUMERICAL RESULTS AND DISCUSSION}

Table 1. Comparison with Newton and Maheshwari method

\section{Example 1}

Consider the equation $f(x)=x^{3}+4 x^{2}-10$.

We start with $x_{n}=2$. The results obtained by Newton iteration, Maheshwari iteration and present the iteration method shown in Tables.

Newton iteration for solving $f(x)=x^{3}+4 x^{2}-10$

\begin{tabular}{c|c|l}
\hline$n$ & $x_{n}$ & \multicolumn{1}{c}{$f\left(x_{n}\right)$} \\
\hline 1 & 1.500000000000000 & 2.375000000000000 \\
\hline 2 & 1.373333333333333 & 0.134345481481482 \\
\hline 3 & 1.365262014874627 & $5.284611795151051 \mathrm{e}-004$ \\
\hline 4 & 1.365230013916147 & $8.290548692002631 \mathrm{e}-009$ \\
\hline 5 & 1.365230013414097 & 0.000000000000000 \\
\hline
\end{tabular}

Maheshwari iteration for solving $f(x)=x^{3}+4 x^{2}-10$

\begin{tabular}{c|c|c}
\hline$n$ & $x_{n}$ & \multicolumn{1}{c}{$f\left(x_{n}\right)$} \\
\hline 1 & 1.383460112875796 & 0.303737459638828 \\
\hline 2 & 1.365230059872621 & $7.671881707693729 \mathrm{e}-007$ \\
\hline 3 & 1.365230013414097 & 0.000000000000000 \\
\hline
\end{tabular}

Proposed iteration for solving $f(x)=x^{3}+4 x^{2}-10$

\begin{tabular}{c|c|c}
\hline$n$ & $x_{n}$ & $f\left(x_{n}\right)$ \\
\hline 1 & 1.366833555991946 & 0.026500759489334 \\
\hline 2 & 1.365230013414097 & 0.000000000000000 \\
\hline
\end{tabular}

\section{Example 2}

Consider the equation, $f(x)=x^{3}-e^{-x}$.

We start with $x_{n}=1$. The results obtained by Newton iteration, Maheshwari iteration and present iteration method is shown in Tables.

Newton iteration for solving $f(x)=x^{3}-e^{-x}$

\begin{tabular}{c|c|l}
\hline$n$ & $x_{n}$ & \multicolumn{1}{c}{$f\left(x_{n}\right)$} \\
\hline 1 & 0.812309030097381 & 0.092166771534313 \\
\hline 2 & 0.774276548985500 & 0.003144824978613 \\
\hline 3 & 0.772884756209622 & $4.050085547491200 \mathrm{e}-006$ \\
\hline 4 & 0.772882959152202 & $6.742550962002269 \mathrm{e}-012$ \\
\hline 5 & 0.772882959149210 & 0.000000000000000 \\
\hline
\end{tabular}

Maheshwari iteration for solving $f(x)=x^{3}-e^{-x}$

\begin{tabular}{c|c|c}
\hline$n$ & $x_{n}$ & \multicolumn{1}{c}{$f\left(x_{n}\right)$} \\
\hline 1 & 0.776281164264182 & 0.007682769461876 \\
\hline 2 & 0.772882959509889 & $8.128708817167762 \mathrm{e}-010$ \\
\hline 3 & 0.772882959149210 & 0.000000000000000 \\
\hline
\end{tabular}

Proposed iteration for solving $f(x)=x^{3}-e^{-x}$

\begin{tabular}{c|c|c}
\hline$n$ & $x_{n}$ & $f\left(x_{n}\right)$ \\
\hline 1 & 0.773074657875942 & $4.321128192329993 \mathrm{e}-004$ \\
\hline 2 & 0.772882959149210 & 0.000000000000000 \\
\hline
\end{tabular}

\section{Example 3}

Consider the equation $f(x)=\sin x$.

We start with $x_{n}=1.5$. The results obtained by Newton iteration, Maheshwari iteration and present iteration method is shown in Tables

Newton iteration for solving $f(x)=\sin x$

\begin{tabular}{c|c|c}
\hline$n$ & $x_{n}$ & $f\left(x_{n}\right)$ \\
\hline 1 & -12.601419947171721 & -0.035042157161019 \\
\hline 2 & 12.566356255118672 & $1.435924050063516 \mathrm{e}-005$ \\
\hline 3 & -12.566370614359174 & $-1.286498119741309 \mathrm{e}-015$ \\
\hline
\end{tabular}

Maheshwari iteration for solving $f(x)=\sin x$

\begin{tabular}{c|c|c}
\hline$n$ & $x_{n}$ & \multicolumn{1}{c}{$f\left(x_{n}\right)$} \\
\hline 1 & -12.140250122502410 & 0.413341334176534 \\
\hline 2 & -12.567623187401553 & -0.001252572714845 \\
\hline 3 & -12.566370614359172 & $4.898587196589413 \mathrm{e}-016$ \\
\hline
\end{tabular}

Proposed iteration for solving $f(x)=\sin x$

\begin{tabular}{c|c|c}
\hline$n$ & $x_{n}$ & $f\left(x_{n}\right)$ \\
\hline 1 & -0.030027696881139 & -0.030023184609464 \\
\hline 2 & $-3.821812658011403 \mathrm{e}-018$ & $-3.821812658011403 \mathrm{e}-018$ \\
\hline
\end{tabular}

\section{Example 4}

Consider the equation $f(x)=\sin x-0.5 x$.

We start with $x_{n}=1.6$. The results obtained by Newton iteration, Maheshwari iteration and present iteration method is shown in Tables. 
Newton iteration for solving $f(x)=\sin x-0.5 x$

\begin{tabular}{c|c|c}
\hline$n$ & $x_{n}$ & $f\left(x_{n}\right)$ \\
\hline 1 & 1.977123551007066 & -0.069983138933437 \\
\hline 2 & 1.898950910895084 & -0.002836729003200 \\
\hline 3 & 1.895501147295299 & $-5.635111423818451 \mathrm{e}-006$ \\
\hline 4 & 1.895494267061370 & $-2.243205621255129 \mathrm{e}-011$ \\
\hline 5 & 1.895494267033981 & 0.000000000000000 \\
\hline
\end{tabular}

Maheshwari iteration for solving $f(x)=\sin x-0.5 x$

\begin{tabular}{c|c|c}
\hline$n$ & $x_{n}$ & \multicolumn{1}{c}{$f\left(x_{n}\right)$} \\
\hline 1 & 1.925586494937329 & -0.025073843759850 \\
\hline 2 & 1.895494852455987 & $-4.794739714153451 \mathrm{e}-007$ \\
\hline 3 & 1.895494267033981 & 0.000000000000000 \\
\hline
\end{tabular}

Proposed iteration for solving $f(x)=\sin x-0.5 x$

\begin{tabular}{c|c|c}
\hline$n$ & $x_{n}$ & $f\left(x_{n}\right)$ \\
\hline 1 & 1.896139437512832 & $-5.286063872829150 \mathrm{e}-004$ \\
\hline 2 & 1.895494267033981 & 0.000000000000000 \\
\hline
\end{tabular}

In Table 2 , we give the number of iterations $(\mathrm{N})$ and the number of function evaluations (NOFE) required to satisfy the stopping criterion, $\mathrm{F}$ denotes that method fails and $\mathrm{D}$ denotes divergence. PM denotes the proposed method. CN denotes the classical Newton method, AN - Arithmetic mean Newton method [18], HN - Harmonic mean Newton method, GN - Geometric mean Newton method [10], MN - Mid- point Newton method.

In Table 3, we give the number of iterations $(\mathrm{N})$ and the number of function evaluations (NOFE) required satisfying the stopping criterion, $\mathrm{F}$ denotes that method fails, D denotes divergence. PM denotes the proposed method. Kou and Li is the method [8]. An improvement of Jarratt method. Gupta denotes for [15] Parhi and Gupta, A sixth order method for nonlinear equations.

In Table 2 and Table 3 the following test functions have been used
(a) $x^{3}+4 x^{2}-10$
$\alpha=1.365230013414097$,
(b) $\sin ^{2} x-x^{2}+1$,
$\alpha=-1.404491648215341$,
(c) $x^{3}-10$,
$\alpha=2.154434690031884$,
(d) $x^{3}-\mathrm{e}^{-x}$,
$\alpha=0.772882959149210$,
(e) $\quad x \sin (1 / x)-0.2 \mathrm{e}^{-x}$,
$\alpha=0.363715708657122$,
(f) $\quad(x-1)^{3}-1$,
$\alpha=2$,
(g) $\quad(x-2)^{23}-1$,
$\alpha=3$,
(h) $\quad x^{2}-\mathrm{e}^{x}-3 x+2$,
$\alpha=0.257530285439861$

Table 2. Comparison with third order methods

\begin{tabular}{|c|c|c|c|c|c|c|c|c|c|c|c|c|c|}
\hline \multirow{2}{*}{$F(x)$} & \multirow{2}{*}{$x_{0}$} & \multicolumn{6}{|c|}{$\mathrm{N}$} & \multicolumn{6}{|c|}{ NOFE } \\
\hline & & $\mathrm{CN}$ & AN & HN & GN & MN & PM & $\mathrm{CN}$ & AN & HN & GN & MN & PM \\
\hline \multirow{2}{*}{ (a) } & -0.5 & 131 & 6 & 65 & $\mathrm{~F}$ & 10 & 6 & 262 & 18 & 195 & - & 30 & 24 \\
\hline & 1 & 5 & 3 & 3 & 3 & 3 & 2 & 10 & 9 & 9 & 9 & 9 & 8 \\
\hline \multirow{2}{*}{ (b) } & -2 & 5 & 5 & 3 & 6 & 3 & 25 & 10 & 15 & 9 & 18 & 9 & 8 \\
\hline & 2 & 5 & 5 & 3 & F & 4 & 2 & 10 & 15 & 9 & $\mathrm{~F}$ & 12 & 8 \\
\hline \multirow{4}{*}{ (c) } & -3 & 18 & $\mathrm{D}$ & 17 & D & $\mathrm{D}$ & 5 & 36 & - & 51 & - & - & 20 \\
\hline & 2 & 4 & 3 & 3 & D & 3 & 2 & 8 & 9 & 9 & - & 9 & 8 \\
\hline & 2.5 & 5 & 3 & 3 & D & 3 & 2 & 10 & 9 & 9 & - & 9 & 8 \\
\hline & 3 & 5 & 4 & 3 & D & 4 & 2 & 10 & 9 & 12 & - & 12 & 8 \\
\hline \multirow{2}{*}{ (d) } & 0.5 & 5 & 4 & 3 & 13 & 3 & 2 & 10 & 12 & 9 & 39 & 9 & 8 \\
\hline & 1 & 5 & 3 & 3 & 15 & 3 & 2 & 10 & 9 & 9 & 45 & 9 & 8 \\
\hline \multirow{3}{*}{ (e) } & 0.2 & 226 & D & 90 & $\mathrm{D}$ & 4 & 5 & 452 & - & 270 & - & 12 & 20 \\
\hline & 0.3 & 3 & 3 & 3 & 29 & 3 & 2 & 9 & 9 & 9 & 8 & 87 & 9 \\
\hline & 0.5 & 5 & 3 & 3 & 29 & 3 & 2 & 10 & 9 & 9 & 87 & 9 & 8 \\
\hline \multirow{2}{*}{ (f) } & 1.8 & 5 & 3 & 3 & 28 & 3 & 2 & 10 & 9 & 9 & 84 & 9 & 8 \\
\hline & 3.5 & 7 & 5 & 4 & 37 & 5 & 3 & 14 & 15 & 12 & 111 & 15 & 12 \\
\hline \multirow{3}{*}{ (g) } & 0.1 & 226 & $\mathrm{D}$ & 90 & $\mathrm{D}$ & 27 & 15 & 452 & - & 270 & - & 81 & 60 \\
\hline & 1 & 232 & D & 79 & D & D & 149 & 464 & - & 237 & - & - & 596 \\
\hline & 3.5 & 14 & 9 & 8 & D & 9 & 6 & 28 & 27 & 24 & - & 27 & 24 \\
\hline \multirow{2}{*}{ (h) } & 2 & 5 & 4 & 4 & D & 3 & 2 & 10 & 12 & 12 & - & 9 & 8 \\
\hline & 3 & 6 & 4 & 4 & 4 & 4 & 3 & 12 & 12 & 12 & 12 & 12 & 12 \\
\hline
\end{tabular}


Table 3. Comparison with sixth order methods

\begin{tabular}{|c|c|c|c|c|c|c|c|}
\hline \multirow{2}{*}{$F(x)$} & \multirow{2}{*}{$x_{0}$} & \multicolumn{3}{|c|}{$\mathrm{N}$} & \multicolumn{3}{|c|}{ NOF E } \\
\hline & & Kou and $\mathrm{Li}$ & Gupta & PM & Kou and $\mathrm{Li}$ & Gupta & PM \\
\hline \multirow{3}{*}{ (a) } & -0.5 & 15 & 62 & 6 & 60 & 248 & 24 \\
\hline & 1 & 2 & 2 & 2 & 8 & 8 & 8 \\
\hline & 2 & 2 & 2 & 2 & 8 & 8 & 8 \\
\hline \multirow{2}{*}{ (b) } & -2 & 2 & 2 & 2 & 8 & 8 & 8 \\
\hline & 2 & 2 & 2 & 2 & 8 & 8 & 8 \\
\hline \multirow{4}{*}{ (c) } & -3 & 4 & 4 & 4 & 16 & 16 & 16 \\
\hline & 2 & 2 & 2 & 2 & 8 & 8 & 8 \\
\hline & 2.5 & 2 & 2 & 2 & 8 & 8 & 8 \\
\hline & 3 & 2 & 2 & 2 & 8 & 8 & 8 \\
\hline \multirow{2}{*}{ (d) } & 0.5 & 2 & 2 & 2 & 8 & 8 & 8 \\
\hline & 1 & 2 & 2 & 2 & 8 & 8 & 8 \\
\hline \multirow{3}{*}{ (e) } & 0.2 & $\mathrm{~F}$ & 13 & 5 & - & 52 & 20 \\
\hline & 0.2 & 2 & 2 & 2 & 8 & 8 & 8 \\
\hline & 0.3 & 2 & 2 & 2 & 8 & 8 & 8 \\
\hline \multirow{2}{*}{ (f) } & 1,8 & 2 & 2 & 2 & 8 & 8 & 8 \\
\hline & 3,5 & 3 & 3 & 3 & 12 & 12 & 12 \\
\hline \multirow{3}{*}{ (g) } & 0.1 & 29 & 118 & 15 & 116 & 476 & 60 \\
\hline & 1 & 180 & 9 & 149 & 720 & 36 & 596 \\
\hline & 3.5 & 5 & 6 & 6 & 20 & 24 & 24 \\
\hline \multirow{2}{*}{ (h) } & 2 & 2 & 2 & 2 & 8 & 8 & 8 \\
\hline & 3 & 2 & 2 & 2 & 8 & 8 & 8 \\
\hline
\end{tabular}

Hence from the Table 1, 2, 3, the main observations are as follows:

1. Present method takes a lesser number of iterations than the others.

2. Example shows that the present method requires a lesser number of functional evaluations, as compared to other methods.

3. The results in the last column of the selected examples, shows the absolute deviation of $f(x)$ in each step with respect to its targeted value, i.e. zero. It can be observed from these examples that the value of absolute error is least for the present method (Table 1, example 3).

Thus, the present method is not only faster but the cost effecting parameters obtain in examples show that it has minimum cost among all the methods taken here.

\section{NUMERICAL RESULTS AND CONCLUSIONS}

In this section, we present the results of some numerical tests to compare the efficiencies of the proposed method. We employed - CN method, Maheshwari method, some third order methods - AN method of Fernando et al. [1], HN, GN, MN methods and some sixth order methods [8] and [15]. Numerical computations reported here have been carried out in MATLAB and the stopping criterion has been taken as $\left|x_{n+1}-\alpha\right|+\left|f\left(x_{n+1}\right)\right|<10^{14}$.

Thus the proposed sixth order method for finding simple real roots of nonlinear equations, is free from second order derivative of the given function, as required in the family of Chebyshev-Halley type methods. Our method requires evaluations of two functions and two first order derivatives per iteration. The convergence analysis of the method is performed in a much simpler way to show that the order of convergence of the method is six. The high order convergence is also corroborated by numerical tests.

The method has the efficiency index equal to 1.5651 , which is better than Newton's method with efficiency index equal to 1.414 and the classical third order methods (1.442), such as Weerakoon and Fernando method, Chebyshev's method, Halley's method and Super-Halley method, fifth order method (1.495) of Kou, Li, Wang [9] and Grau, Noguera [4] and sixth order method (1.565) of Parhi and Gupta [15] and Kou and Li [8]. The method is tested on a number of numerical examples. On comparing our results with those obtained 
by Newton's method (NM) third order methods and fourth order methods, it is found that our method is most effective as it converges to the root much faster. When compared with the sixth order methods of Parhi, Gupta, [15] and Kou and Li [8], our method behaves either similarly or better on the examples considered.

\section{References}

[1] C. Chun, Some improvements of Jarratt's method with sixth-order convergence. Appl. Math. Comput. 190, 14321437 (2007).

[2] J. Chen, Some new iterative methods with three-order convergence. Appl. Math. Comput. 181, 1519-1522 (2006).

[3] M. Frontini and E. Sormani, Some variant of Newton's method with third-order convergence. Appl. Math. Comput. 140, 419-426 (2003).

[4] M. Grau and M. Noguera, A variant of Cauchy's method with accelerated fifth-order convergence. Appl. Math. Lett. 17, 509-517 (2004).

[5] M.K. Jain, S.R.K. Iyengar and R.K. Jain, Numerical Methods for Scientific and Engineering Computation. New York, NY: Halsted Press, 1985.

[6] J. Kou and X. Wang, Sixth-order variants of ChebyshevHalley methods for solving non-linear equations. Appl. Math. Comput. 190, 1839-1843 (2007).

[7] J. Kou, On Chebyshev-Halley methods with sixth-order convergence for solving non-linear equations. Appl. Math. Comput. 190, 126-131 (2007).
[8] J. Kou and Y. Li, An improvement of Jarratt method. Appl. Math. Comput. 189, 1816-1821 (2007).

[9] J. Kou, Y. Li, and X. Wang, Some modifications of Newton's method with fifth order convergence. Journal of Computational and Applied Mathematics 209, 146152 (2009).

[10] T. Lukic and N.M. Ralevie, Geometric mean Newton's method for simple and multiple roots. Applied Mathematics Letters 21, 30-36 (2008).

[11] A.K. Maheshwari, A fourth-order iterative method for solving non-linear equations. Applied Mathematics and Computation 211, 383-391 (2009).

[12] A.M. Ostrowski, Solution of Equations and Systems of Equations. Academic Press Inc., 1966.

[13] J.M. Ortega and W.C. Rheinboldt, Iterative Solution of Nonlinear Equations in Several Variables. Academic Press, New York, 1970.

[14] A.Y. Ozban, Some new variants of Newton's method. Applied Mathematics Letters 17, 677-682 (2004).

[15] S.K. Parhi and D.K. Gupta, A sixth order method for nonlinear equations. Applied Mathematics and Computation 203, 50-55 (2008).

[16] J.R. Sharma and R.K. Guha, A family of modified Ostrowski methods with accelerated sixth order convergence. Appl. Math. Comput. 190, 111-115 (2007).

[17] J.F. Traub, Iterative Methods for the Solution of Equations. Prentice Hall, Clifford, NJ, 1964.

[18] S. Weerakoon and T.G.I. Fernando, $A$ variant of Newton's method with accelerated third-order convergence. Appl. Math. Lett. 13, 87-93 (2000).

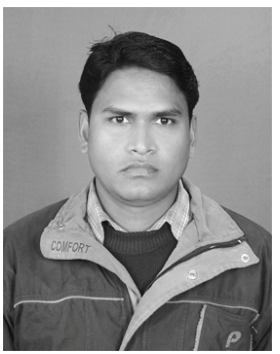

ManoJ Kumar Singh is born in Holy city Varanasi of India on 15 July 1982. His nationality is Indian. He won the N B H M Scholarship (All India Scholarship) for M.Sc. during 2004. In that year, he received M.Sc. from the Department of Mathematics, Banaras Hindu University, Varanasi, India. Currently he is a PhD student under the guidance of prof S. R. Singh, at the Department of Mathematics of Faculty of Science, Banaras Hindu University, Varanasi, India. His professional interests concern unconstrained optimization and its modelling. 